Range (ft.) $\quad 300-500 \quad 500-700 \quad 700-900 \quad 900-1,100 \quad 1,100-1,200$ Gravi-

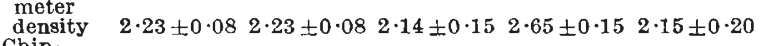

Chip-

pings

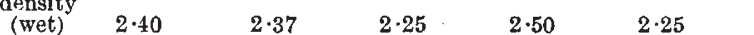

$\begin{array}{llllll}\text { (wet) } & 2 \cdot 40 & 2 \cdot 37 & 2 \cdot 25 & 2 \cdot 50 & 2 \cdot 25 \\ \text { (dry) } & 2 \cdot 25 & 2 \cdot 17 & 1 \cdot 97 & 2 \cdot 33 & 2 \cdot 0\end{array}$

should not be drawn, however, as the effect of assuming a linear drift of the 'zero' frequency is to indicate a lower density than would be obtained if the alternative drift curve were adopted.

I should like to thank the Anglo-Iranian Oil Co., Ltd., for financial support during the period of research, for permission to publish this communication and for allowing me to use their borehole and for providing a suitable cable and winch for the experiments. I should also like to thank the chairman and members of the Paul. Instrument Fund Committee for a grant enabling the gravimeter to be built, Prof. A. O. Rankine for his interest in the work, and Mr. B. C. Browne and Dr. R. I. B. Cooper, of the Department of Geodesy and Geophysics, Cambridge, for their help and encouragement during the investigation.

Department of Geodesy and Geophysics,

R. L. G. GILbert

Downing Place, Cambridge. June 18.

${ }^{1}$ Smith, Geophysics, 15, No. 4, 605 (1950).

${ }^{2}$ Gilbert, Proc. Phys. Soc., B, 62, 445 (1949).

\section{Electromagnetic Momentum and Newton's Third Law}

THE following simple relations ${ }^{1}$ between electromagnetic momentum, Poynting's vector of energy flow, and mass-energy equivalence do not appear to have received general recognition in connexion with the validity of Newton's Third Law when applied to electromagnetic forces.

If the dielectric medium is free space, so that there is no matter present to take reactive forces, we suppose that the energy represented by the Poynting vector, $\vec{\varepsilon} \times \mathbf{H}$ (rationalized M.K.S. units), moves with the velocity of light and also has mass of amount $m$ per unit volume of the field. Suppose also that its momentum per unit volume is $\mathbf{D} \times \mathbf{B}$ (where $\mathbf{D}=\varkappa_{0} \vec{\varepsilon}$, $\left.\mathbf{B}=\mu_{0} \mathbf{H}, \varkappa_{0} \mu_{0}=1 / c^{2}\right)$, and consider a unit area of surface normal to the direction of energy flow. Then if the rate at which energy passes through this surface is $|\vec{\varepsilon} \times \mathbf{H}|$, it follows that the amount of moving energy per unit volume of the field is $W=\vec{\varepsilon} \times \mathbf{H} \mid / c$. Its momentum is $m c$, so if this is to be equal to $|\mathbf{D} \times \mathbf{B}|$, we must have :

$$
m=\frac{|\mathbf{D} \times \mathbf{B}|}{c}=\frac{|\vec{\varepsilon} \times \mathbf{H}|}{c^{3}}=\frac{W}{c^{2}},
$$

which is the mass-energy relation.

Whenever the total 'ponderomotive' force on an electromagnetic system is not zero, it is equal to the volume integral of $-\frac{\partial}{\partial t}(\mathbf{D} \times \mathbf{B})$ taken over the whole of the dielectric in which $\mathbf{D}$ and $\mathbf{B}$ exist. It is therefore balanced by the volume integral of $\frac{\partial}{\partial t}(\mathbf{D} \times \mathbf{B})$, the time-rate of change of the total electromagnetic momentum. The interpretation is clearly that this represents the rate of change of ordinary mass-momentum of the energy which is moving about in the changing field, and that the 'equal and opposite' reaction to the unbalanced ponderomotive force is borne by the energy photons.

Newton's Third Law is thus reinstated if the mass of the field energy is taken into account. The theorem applies both to radiating and to non-radiating systems. A simple example of the latter is to be found in the case of a charged particle moving with uniform velocity through a constant-current toroidal coil.

University College,

E. G. Cullwick

Dundee. March 21

1 See, for example, Lorentz, H. A., "Lectures on Theoretical Physics", 241 and 249 (English trans., Macmillan, 1931), in the (hapter on "The Inertia of Energy".

\section{Screw Dislocations in Pyrite}

$I_{N}$ the course of an investigation into the surface structure of a wide range of mineral crystals, the results of which were reported to the Mineralogical Society in March 1951, a number of screw dislocations were observed on the cube faces of one crystal of pyrite, $\mathrm{FeS}_{2}$. Fig. 1 shows a single screw dislocation; while Fig. 2 exhibits a pair of screw dislocations of opposite sense, which ultimately form an almost closed step-line. The crystal was photographed using a metallurgical microscope, with bright-field illumination, and the surfaces were not silvered. Under these conditions, the very high visibility of the steps forming the main spiral patterns indicates that the layers are multimolecular. This point is further substantiated by the presence of some much thinner layers between the main steps. Examples of screw dislocations with a Burgers vector greater than unity, often considerably greater, have already been reported for cadmium iodide ${ }^{1}$, silicon carbide $^{2}$, and muscovite ${ }^{3}$.

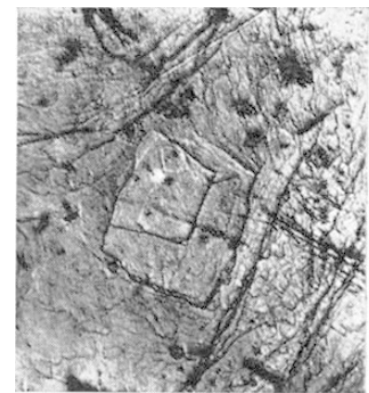

Fig. $1(\times 35)$

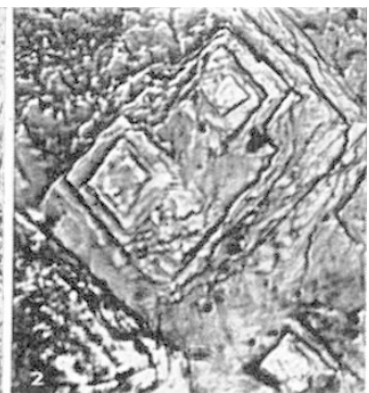

Fig. $2(\times 35)$
The incompleteness of the spiral patterns in Fig. 2 appears to be due to the separation of the thickest layers into several thinner ones in some places.

Note added in proof. A paper on the surface structure of crystals is to be published in the Mineralogical Magazine.

Department of Geology

A. F. Seager Birkbeck College,

University of London:

Malet Street, W.C.1. July 8.

${ }^{2}$ Forty, A. J., Phil. Mag., 42, 670 (1951).

2 Verma, A. R., Nature, 188, 430 (1951).

${ }^{3}$ Amelinckx, S., Nature, 168, 580 (1952). 